\title{
A comparison of tandem ring and tandem ovoid treatment as a curative brachytherapy component for cervical cancer
}

\author{
Sukriye Bilge Gursel, MD, Assoc. Prof., Alparslan Serarslan, MD, Ahmet Deniz Meydan, MD, Assoc. Prof., \\ Prof. Nilgun Okumus, MD, Tenzile Yasayacak, Phys. \\ Radiation Oncology Department, School of Medicine, Ondokuz Mayis University, Samsun, Turkey
}

\begin{abstract}
Purpose: The standard treatment for locally advanced stage cervical cancer is definitive radiotherapy, the quality of which affects both survival and side effects. Brachytherapy is a major component of definitive radiotherapy; it is administered using different techniques and applicators. The purpose of this study was to dosimetrically compare tandem ovoid (T-ovoid) and tandem ring (T-ring) brachytherapy treatments.

Material and methods: Both applicator sets were applied to the same 20 patients, and treatment plans were made three-dimensionally (3D), with high-risk clinical target volume (HR-CTV) and organs at risk contoured. The HR-CTV was defined according to post-external magnetic resonance results. The patients with residual tumors not exceeding one-third of the parametrium were included in this study, while patients with larger masses were excluded and received interstitial therapy. The doses were calculated for both plans. Optimization for the HR-CTV was made with the aim that the equivalent dose according to $2 \mathrm{~Gy}\left(\mathrm{EQD}_{2}\right)$ of $90 \%$ of the HR-CTV $\left(\mathrm{D}_{90}\right)$ would be higher than $85 \mathrm{~Gy}$, without exceeding the maximum dose for organs at risk. Then, pairwise dosimetric comparisons were performed.

Results: Plans were compared dosimetrically according to the HR-CTV, point A and B doses, and organs at risk. Although the point A and B doses were higher with T-ovoid use, the 3D HR-CTV coverage was statistically better with T-ring application $\left(\mathrm{EQD}_{2}\right.$ of HR-CTV $\mathrm{D}_{90}$ : $97.46 \mathrm{~Gy}$ for T-ring and $88.44 \mathrm{~Gy}$ for T-ovoid; $\left.p<0.0001\right)$. In addition, the rectum and bladder doses were statistically lower with T-ring usage $\left(\mathrm{EQD}_{2}\right.$ of rectum, $2 \mathrm{cc}$; T-ring, 63.10 Gy; T-ovoid, 74.99 Gy; $p<0.0001 ; \mathrm{EQD}_{2}$ of bladder, 2 cc; T-ring, 85.26 Gy; T-ovoid, $89.05 \mathrm{~Gy} ; p<0.0001$ ).

Conclusions: In our study with a limited number of samples, T-ring applicator seems to offer better 3D brachytherapy dosimetry for both HR-CTV and nearby organs at risk.

Key words: brachytherapy, ring, ovoid, three-dimensional planning, high-risk clinical target volume.

\section{Purpose}

Cervical cancer is the fourth most common cancer worldwide and the most common gynecologic cancer $[1,2]$. In Turkey, cervical cancer was reported to be the third most common gynecologic cancer after corpus uteri and ovarian carcinoma, and the ninth most common malignancy in women [3].

Cervical cancer is typically diagnosed at the locally advanced stage (> 80\%; Fédération Internationale de Gynécologie et d'Obstétrique - FIGO), generally at stage IB2IVA and/or node-positive stages in developing countries such as Turkey $[3,4]$. The standard treatment for patients with locally advanced cervical cancer is definitive radiotherapy, including concomitant external chemoradiother- apy and brachytherapy. The quality of definitive radiotherapy both increases loco-regional control rates and decreases side effects $[5,6,7]$. Although the Manchester system has been widely used with these treatments, the target area coverage and radiation risk to nearby tissues are still uncertain [8].

Three-dimensional (3D) brachytherapy has shown trends of increased local tumor control and overall survival with reduced toxicity, compared with the conventional two-dimensional brachytherapy technique [9]. A few studies have demonstrated that the quality of brachytherapy depends on the application technique used $[10,11,12,13]$.

The most commonly used type of applicator for curative definitive cervical cancer brachytherapy is the tan-

Address for correspondence: Sukriye Bilge Gursel, MD, Assoc. Prof., Radiation Oncology Department, Received: 08.08 .2019 School of Medicine , Ondokuz Mayis University, Kurupelit 55270, Samsun, Turkey, phone: +90 5058002030, Accepted: 29.02.2020 
dem ovoid (T-ovoid) applicator, followed by the tandem ring (T-ring) applicator. T-ovoid treatment offers a range of sizes and allows for configuration adjustment, whereas the advantages of T-ring application include the different loading positions and repeatability due to its compact configuration. However, there are insufficient data to determine which applicator type offers superior radiation delivery and protection, especially when using a 3D technique. Therefore, we performed a dosimetric comparison of targets (point doses and volumetric) and organs at risk according to applicator in patients who were treated with 3D brachytherapy using both applicator types.

\section{Material and methods}

This study was approved by the local ethics committee of the Ondokuz Mayis University Hospital in Samsun, Turkey (protocol \#2019/352), and all patients provided informed consent prior to treatment. For this prospective clinical trial, a minimum of 13 patients in each group was required to identify a difference in rectal dose, with a power of $80 \%$ at the two-sided $5 \%$ significance level, according to the report of Ma et al. [13] (which calculated a mean rectal dose of $2.23 \pm 0.65 \mathrm{~Gy}$ with the T-ovoid applicator, and $1.62 \pm 0.35$ Gy with the T-ring applicator; $p=0.0018$ ). However, to identify differences in all other parameters, we needed to involve additional patients. Given our limited number of patients, we included 20 plans in each group.

T2-weighted magnetic resonance imaging (MRI) images were obtained prior to brachytherapy to determine the extent of residual disease, and to guide the delineation of high-risk clinical target volume (HR-CTV). This study included 20 patients, whose residual tumors did not exceed one-third of the parametrium following external chemoradiotherapy, as shown on T2-weighted MRI images. Patients with greater residual tumor extension were excluded from the current study but received interstitial treatment.

Patients were advised to start using a laxative 2 days before the scheduled treatment. In our clinic, we do not routinely use an enema. After sedation was induced, each patient was placed in the dorsal lithotomy position, and a Foley catheter was inserted. The balloon was inflated with 7 cc contrast and, after emptying the bladder, it was filled with a 150-cc contrast-saline combination (1:10). The Foley catheter was plugged to keep the bladder full, thereby decreasing anteversion of the uterus. Following gynecological examination and measurement of the uterine cavity, a T-ring applicator with rectal retractor was inserted by a radiation oncologist. The brachytherapy system was a GammaMed Plus HDR device, which contained ${ }^{192} \mathrm{Ir}$; both applicator sets were Varian sets (Varian Medical Systems, Palo Alto, CA, USA).

Computerized tomography (CT; Asteion Super 4, Toshiba Medical Systems Co. Ltd., Otawara, Japan) with a thickness of $3 \mathrm{~mm}$ was conducted. The same radiation oncologist performed contouring according to the Groupe Européen de Curiethérapie-European Society for Radiotherapy and Oncology (GEC-ESTRO) guidelines for contouring, image-based treatment planning and dose reporting $[14,15,16]$. A radiation oncologist contoured the
HR-CTV (by reference to the MRI findings), which included a residual tumor following external radiotherapy, the entire cervix, and the presumed extra-cervical tumor extension at the time of brachytherapy. No clear definition of the HR-CTV was provided in previous studies on CT-based planning. Many clinics, including ours, cannot always conduct post-applicator MRI planning. In addition, as reported in previous studies, brachytherapy with CT planning with or without pre-brachytherapy MRI, has been used frequently. Our protocol stipulated the performance of a pre-brachytherapy MRI study; if a tumor exceeding one-third of the length of the parametrium was present, the patients were excluded from the study and received interstitial implants. We used CT imaging and pre-brachytherapy MRI findings to define the HR-CTV.

The radiation oncologist defined the bladder, rectum, and sigmoid as organs at risk, according to standard protocols. The second intervention was performed by placing a T-ovoid applicator by the same radiation oncologist. We used the largest ovoid that could be inserted with the tandem, the same size as the T-ring applicator. Vaginal packing was performed, and an additional CT scan (3-mm slice thickness) was performed. The HR-CTV and organs at risk were contoured in a similar manner. This study was not conducted as an adaptive therapy. Therefore, we used the same residual disease burden determined on post-external radiotherapy MRI images for the HR-CTV contouring, and the same radiation oncologist contoured the second CT image (Figure 1). Simulation and planning were conducted during the first two brachytherapy sessions. The BrachyVision planning system (v. 13.7; Varian Medical Systems, Palo Alto, CA, USA) utilizing the calculation algorithm TG-43 was used for planning and calculation. Dose-volume histogram (DVH) parameters were used for optimization and approval. All treatment plans were determined by the same radiation oncologist and medical physicist, according to the gynecological GEC-ESTRO guidelines $[14,15,16]$. A dose of 6 Gy was initially prescribed for the HR-CTV, and manual optimization was conducted, which did not exceed the GEC-ESTRO guidelines for organs at risk. All dwell positions were activated initially. Next, with the help of a isodose shaper tool and based on the dwell time changes, the isodose was modified and manual modifications were made to maintain the pear-shaped dose distribution, while excluding the rectum, sigmoid, and bladder from the final optimized plan. Then, the plans were checked by calculating an equivalent dose in $2 \mathrm{~Gy}$ fractions $\left(\mathrm{EQD}_{2}\right)$ to $\mathrm{D}_{90}$ of $>85 \mathrm{~Gy}$ (assuming that the patient was being treated with a given applicator in five fractions). For organs at risk, the doses recommended by the GEC-ESTRO were as follows: rectum, $\mathrm{D}_{2 \mathrm{cc}}<75$ Gy; sigmoid, $\mathrm{D}_{2 \mathrm{cc}}<75 \mathrm{~Gy}$; and bladder, $\mathrm{D}_{2 \mathrm{cc}}$ $<90$ Gy [8,9]. When the recommended doses could not be achieved, the $\mathrm{D}_{90}$ was lowered to $80 \mathrm{~Gy}$ in the early stage small HR-CTV volume patients and in two patients, the use of a second type of applicator achieved a better HRCTV. A higher bladder dose was accepted for more locally advanced patient (in whom the bladder $\mathrm{D}_{2 \mathrm{cc}}$ was high for both applicator types), after obtaining an informed consent. We tried to lower the dose by changing the bowel 


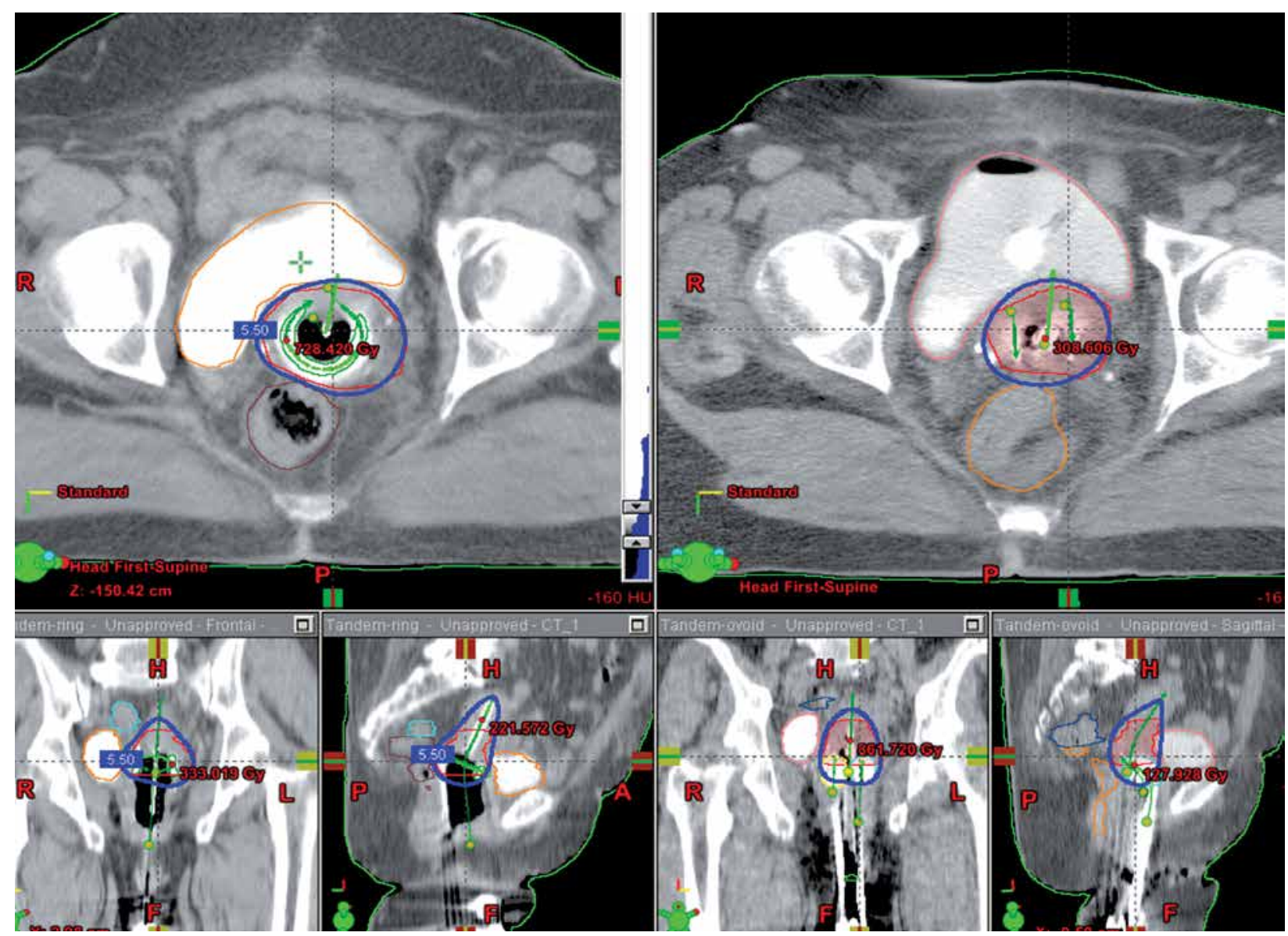

Fig. 1. T-ring (right side, three images) and T-ovoid (left side, three images) applications and plans in the same patient: axial, coronal, and sagittal planes images (images used with a permission from the patient)

preparation method and bladder filling procedure during the remaining three treatment sessions (Figure 1).

Right and left A and B points were determined, and their doses recorded according to the International Commission on Radiation Units and Measurements (ICRU)38 for comparison in both planes [17]. The remaining 3D brachytherapy application was performed according to the two planes, whichever was better (16 patients with T-ring, and four patients with T-ovoid). The radiation oncologist preferred a T-ring with more rigid design for the first brachytherapy session and therefore, prevented its application in some cases $(n=2)$. Thus, these patients were excluded from the study, and treated with the T-ovoid applicator.

Dosimetric comparisons were made in cases where both types of applicator were used. The first applications were made with a T-ring, and the second with a T-ovoid. Following dosimetric comparison of the two applications, the remaining last three applications were made by the applicator that was found to be superior. The plans were not compared at the time of treatment, due to possible extension of planning time; we did not plan to remove one applicator and insert the other one during the same day. The plans were designed to be suitable for independent use.

The total EQD 2 applied to tumors and organs at risk was calculated as the sum of planned pelvic midline ex- ternal beam radiotherapy dose and the equivalent dose from brachytherapy. The pelvic midline external radiotherapy doses were 45 Gy and 50.4 Gy, with a median of $45 \mathrm{~Gy}$. An external radiotherapy dose exceeding 50.4 Gy in the midline was not permitted, because it results in lower dose brachytherapy and an insufficient total dose. In three patients, the low pelvic and parametrial boost doses were delivered in anterior posterior directions to protect the midline. There were $10 \mathrm{lymph}$ node boost doses, with the boost fields situated outside the midline. The boost doses to the parametrium and lymph nodes were not included in the total dose for the $\mathrm{EQD}_{2}$ calculations, and for the HR-CTV and organs at risk. For the $\mathrm{EQD}_{2}$ calculations, the patient was assumed to receive the dose in five fractions, where the dose was calculated using a standard equation with an $\alpha / \beta$ of $10 \mathrm{~Gy}$ for tumors and $3 \mathrm{~Gy}$ for organs at risk.

The SPSS Statistical Package was used for all statistical analyses (v.15.0; SPSS Inc., Chicago, IL, USA). Data normality was evaluated using the Shapiro-Wilk test and, for non-normally distributed data, the non-parametric Wilcoxon test.

\section{Results}

This study included 20 patients with cervical cancer who would benefit from curative brachytherapy and had 
tumors not exceeding one-third of the parametrium after external radiotherapy. Two patients were excluded from the protocol due to an inability to accommodate the applicator's size and fixed configuration. The median patient age was 55 (range, 31-75) years, and the diagnosis was squamous cell carcinoma in 19 patients and adenosquamous cell carcinoma in 1 patient. Of the 20 patients, 4 were diagnosed with stage IB2, 11 with stage IIB, and with 5 stage IIIB. At the time of diagnosis, 10 patients had positive lymph nodes on positron emission tomography $\mathrm{CT}$, and 16 had parametrial involvement. The median pelvic external radiotherapy dose was 45 (range, 45-50.4 Gy). Ten lymph node boost doses (range, 5.4-12 Gy) and three midline blocked doses (45 Gy pelvic, then 5.4-9 Gy) were applied. The HR-CTV was similar between the groups: $28.54 \mathrm{~cm}^{3}$ for the T-ring, and $28.39 \mathrm{~cm}^{3}$ for the T-ovoid $(p=0.17)$. The contoured volumes of organs at risk were similar between the applicators. The bladder volumes were 194.75 and $200.50 \mathrm{~cm}^{3}(p=0.28)$, the rectum volumes were 63.55 and $59.80 \mathrm{~cm}^{3}(p=0.78)$, and the sigmoid vol- umes were 44.40 and $55.05 \mathrm{~cm}^{3}(p=0.71)$, with use of the T-ring and T-ovoid applicators, respectively.

Although point A and B doses were high in the T-ovoid group (Table 1), the volumetric coverage of the HR-CTV was significantly better with T-ring administration (median $\mathrm{EQD}_{2}$ to HR-CTV $\mathrm{D}_{90}, 97.46 \mathrm{~Gy}$ for T-ring, and $88.44 \mathrm{~Gy}$ for T-ovoid; $p<0.0001$ ). The volume that was covered by a $6 \mathrm{~Gy} /$ fraction dose was $97.65 \%$ with T-ring usage, but only $92.10 \%$ with T-ovoid application $(p<0.0001)$. Dose-volume comparisons revealed better coverage by the T-ring applicator (Table 2 and Figure 2). Regarding organs at risk, the $\mathrm{EQD}_{2}$ to the rectum (2 cc volume) was maintained at $63.09 \mathrm{~Gy}$, with a $<3 \%$ risk of grade 1-3 toxicities, with T-ring application. However, the $\mathrm{EQD}_{2}$ was maintained at $74.99 \mathrm{~Gy}$ with the T-ovoid application $(p<0.0001)$. Bladder protection was also statistically better with T-ring application, with no difference in the sigmoid dose. The $\mathrm{EQD}_{2}$ to the bladder $(2 \mathrm{cc}$ volume) was 85.26 and 89.05 Gy with T-ring and T-ovoid applications, respectively $(p<0.0001)$ (Table 3).

Table 1. Fraction and total equivalent total doses: comparison of point doses between the T-ring and T-ovoid applicators. In the calculations of equivalent total dose, it was assumed that the dose was delivered in five fractions

\begin{tabular}{lcccc} 
Point doses & Gy & $\begin{array}{c}\text { T-ring } \\
\text { Median (min-max })\end{array}$ & $\begin{array}{c}\text { T-ovoid } \\
\text { Median (min-max })\end{array}$ & $P$-value \\
\hline Right A point & Dose/fx & $5.11(4.30-5.41)$ & $5.43(5.01-6.30)$ & $<0.0001$ \\
\cline { 2 - 4 } & $\mathrm{EQD}_{2}$ & $77.25(76.02-84.65)$ & $79.91(76.33-93.19)$ & $<0.0001$ \\
\hline Left A point & Dose/fx & $5.22(4.00-5.99)$ & $5.61(5.00-6.48)$ & $<0.0001$ \\
\hline & $\mathrm{EQD}_{2}$ & $78.14(73.73-90.31)$ & $81.88(76.25-94.90)$ & $<0.0001$ \\
\hline Right B point & Dose/fx & $1.30(1.05-1.46)$ & $1.47(1.13-1.81)$ & 0.003 \\
\hline Left B point & $\mathrm{EQD}_{2}$ & $51.30(49.83-57.32)$ & $52.37(50.24-58.91)$ & 0.002 \\
\hline & $\mathrm{Dose}_{\mathrm{fx}}$ & $1.27(1.01-1.73)$ & $1.42(1.08-1.79)$ & 0.001 \\
\hline
\end{tabular}

$f x$-fraction, $E Q D_{2}$-total equivalent dose for 2 Gy fractionated dose, min-max-minimum-maximum

Table 2. Fraction and equivalent total doses: comparison of high-risk clinical target volumes between the T-ring and T-ovoid applicators. In the calculations of equivalent total dose, it was assumed that the dose was delivered in five fractions

\begin{tabular}{|c|c|c|c|c|}
\hline HR-CTV & Gy & $\begin{array}{c}\text { T-ring } \\
\text { Median (min-max) }\end{array}$ & $\begin{array}{c}\text { T-ovoid } \\
\text { Median (min-max) }\end{array}$ & $P$-value \\
\hline \multirow[t]{2}{*}{$\mathrm{D}_{98}$} & Dose/fx & $5.95(4.57-6.73)$ & $5.26(3.70-5.66)$ & $<0.0001$ \\
\hline & $\mathrm{EQD}_{2}$ & 86.57 (72.74-94.40) & $80.88(66.12-85.17)$ & $<0.0001$ \\
\hline \multirow[t]{2}{*}{$D_{95}$} & Dose/fx & $6.47(5.09-7.37)$ & $5.70(4.08-6.15)$ & $<0.0001$ \\
\hline & $\mathrm{EQD}_{2}$ & $91.18(77.00-100.08)$ & 85.46 (68.94-90.09) & $<0.0001$ \\
\hline \multirow[t]{2}{*}{$D_{90}$} & Dose/fx & $7.09(5.70-8.20)$ & $6.21(4.60-7.15)$ & $<0.0001$ \\
\hline & $\mathrm{EQD}_{2}$ & $97.46(82.29-107.64)$ & 88.44 (77.98-96.09) & $<0.0001$ \\
\hline \multicolumn{5}{|c|}{ Volume \% } \\
\hline$V_{5.5 G y}$ & & $99.2 \%(91.80-100.00)$ & $96.4 \%(91.20-98.70)$ & 0.001 \\
\hline $\mathrm{V}_{6 \mathrm{~Gy}}$ & & $97.65 \%$ (87.10-99.65) & $92.10 \%(86.90-95.92)$ & $<0.0001$ \\
\hline
\end{tabular}

HR-CTV - high-risk clinical target volume, $V_{x G y}$-volume percent that gets a dose of $x$ Gy or higher, $D_{x}$-the dose that is absorbed by $x \%$ of the volume, $f x$ - fraction, $E Q D_{2}$ - total equivalent dose for 2 Gy fractionated dose, min-max - minimum-maximum 


\section{Discussion}

The reported median age at diagnosis of cervical cancer in the U.S. is approximately $47-48$ years and is even lower in human papillomavirus (HPV)-positive patients [18]. In the Retro-EMBRACE study, the median age at diagnosis was 53 (range, 23-91) years [7]. In Turkey, the HPV-positive population is thought to be smaller than the worldwide average, but there are no statistical data supporting this conclusion. In our study, the median patient age was 55 (range, 31-75) years.

Higher radiotherapy doses for chemoradiotherapy and brachytherapy for advanced cervical cancer have been shown to improve local tumor control $[5,6]$. Recently, the EMBRACE I study showed that $98 \%$ of local recurrences were seen within intermediate-risk CTVs and HR-CTVs [19]. The Retro-EMBRACE study also revealed correlations of local tumor control with volume, dose, and total treatment time [20]. Fokdal et al. [21] suggested adding interstitial brachytherapy for treatment of large HR-CTV tumors with diffuse parametrial involvement, and they reported a 3-year local control rate of $93 \%$ for tumors with a medium-sized HR-CTV $\left(20-30 \mathrm{~cm}^{3}\right)$, when a $\geq 85$ Gy dose was applied. In our study, the median HR-CTV was $28 \mathrm{~cm}^{3}$.

For medium and small-sized HR-CTVs, there is no clear consensus regarding which applicator type should be used. Generally, the most commonly used applicators

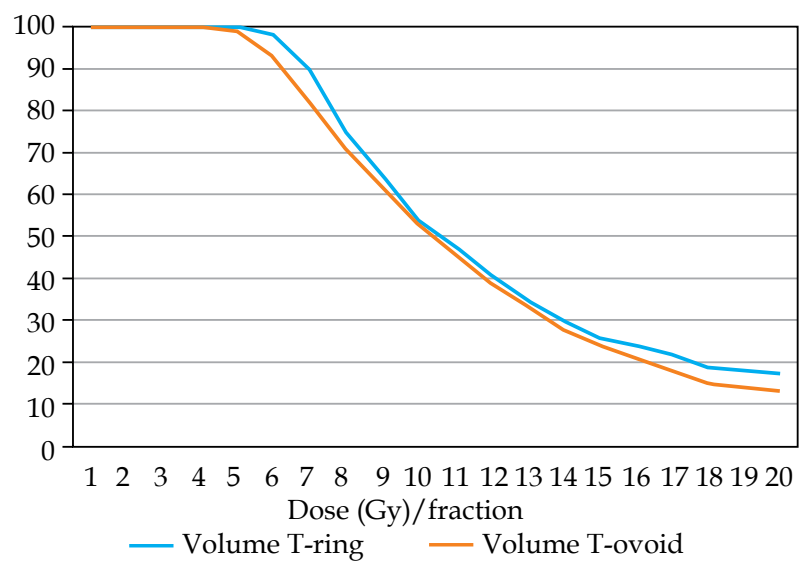

Fig. 2. Dose-volume histogram of the high-risk clinical target volume using the T-ring and T-ovoid applicators (one fraction). The mean doses to $100 \%, 99 \%, 97 \%, 95 \%, 90 \%$, $80 \%, 70 \%, 60 \%, 50 \%, 40 \%, 30 \%, 20 \%$, and $10 \%$ volumes were calculated in each group, and a cumulative dose-volume histogram was obtained. Blue: T-ring group; orange: T-ovoid group

are the T-ovoid (with vaginal packing) and T-ring (with a rectal retractor). The applicator type is usually chosen according to tumor size and anatomy of the patient. The T-ring has a rigid design and is preferred because of the

Table 3. Fraction and total equivalent total doses: comparison of doses to organs at risk between the T-ring and T-ovoid applicators. In the calculations of equivalent total dose, it was assumed that the dose was delivered in five fractions

\begin{tabular}{|c|c|c|c|c|}
\hline Organ at risk & Gy & $\begin{array}{c}\text { T-ring } \\
\text { Median (min- max) }\end{array}$ & $\begin{array}{c}\text { T-ovoid } \\
\text { Median (min-max) }\end{array}$ & $P$-value \\
\hline \multirow[t]{2}{*}{ Bladder 2cc } & Dose/fx & $5.02(4.38-5.35)$ & $5.17(4.48-5.48)$ & $<0.0001$ \\
\hline & $\mathrm{EQD}_{2}$ & 85.26 (77.32-90.39) & 89.05 (78.51-91.71) & $<0.0001$ \\
\hline \multirow[t]{2}{*}{ Bladder 1cc } & Dose/fx & $5.40(4.87-5.96)$ & $5.82(5.17-6.34)$ & $<0.0001$ \\
\hline & $\mathrm{EQD}_{2}$ & $90.42(83.33-98.40)$ & $96.48(87.24-104.70)$ & $<0.0001$ \\
\hline \multirow[t]{2}{*}{ Bladder 0.1cc } & Dose/fx & $5.82(5.17-6.34)$ & 6.34 (5.59-7.98) & 0.002 \\
\hline & $\mathrm{EQD}_{2}$ & $106.20(99.73-132.62)$ & $121.24(106.67-175.23)$ & 0.003 \\
\hline \multirow[t]{2}{*}{ Rectum 2cc } & Dose/fx & $2.82(2.07-3.85)$ & 3.89 (3.64-4.23) & $<0.0001$ \\
\hline & $\mathrm{EQD}_{2}$ & 63.09 (55.49-74.79) & 74.99 (69.17-75.84) & $<0.0001$ \\
\hline \multirow[t]{2}{*}{ Rectum 1cc } & Dose/fx & $3.08(2.31-4.42)$ & $4.45(4.29-5.37)$ & $<0.0001$ \\
\hline & $\mathrm{EQD}_{2}$ & $65.43(57.27-82.80)$ & 83.04 (76.27-89.95) & $<0.0001$ \\
\hline \multirow[t]{2}{*}{ Rectum 0.1cc } & Dose/fx & $3.71(2.86-5.84)$ & $5.53(5.28-6.35)$ & $<0.0001$ \\
\hline & $\mathrm{EQD}_{2}$ & 72.37 (61.76-97.31) & 93.71 (88.72-102.04) & 0.001 \\
\hline \multirow[t]{2}{*}{ Sigmoid 2cc } & Dose/fx & 2.87 (1.38-3.99) & $2.39(1.33-3.76)$ & 0.071 \\
\hline & $\mathrm{EQD}_{2}$ & 61.85 (51.04-73.85) & $59.24(50.76-75.42)$ & 0.112 \\
\hline \multirow[t]{2}{*}{ Sigmoid 1cc } & Dose/fx & $3.29(1.52-4.89)$ & $2.82(1.45-4.82)$ & 0.218 \\
\hline & $\mathrm{EQD}_{2}$ & $65.88(51.87-88.60)$ & $61.41(51.45-88.09)$ & 0.247 \\
\hline \multirow[t]{2}{*}{ Sigmoid 0.1cc } & Dose/fx & $4.38(1.82-6.42)$ & $3.37(1.65-6.91)$ & 0.092 \\
\hline & $\mathrm{EQD}_{2}$ & 77.38 (53.77-105.48) & $66.54(52.67-118.88)$ & 0.179 \\
\hline
\end{tabular}

$f x$-fraction, $E Q D_{2}$-total equivalent dose for 2 Gy fractionated dose, min-max-minimum-maximum 
repeatability of applications; however, its inflexibility sometimes prevents insertion. T-ovoid applicators can be inserted individually, and more sizes are available. In our study, we were able to treat two patients with T-ovoid applicators, in whom T-ring applicators could not be inserted.

In our study, for patients in whom both applicators could be inserted, the $\mathrm{D}_{90}$ of the HR-CTV was $88.44 \mathrm{~Gy}$ with the T-ovoid applicator, and was approx. 9 Gy higher, at $97.46 \mathrm{~Gy}$, with the T-ring applicator. Since both applicator types were applied to the same patient group, therefore, it was not possible to evaluate survival or side effect differences. In our study, the rectum was defined as an organ at risk, when the $\mathrm{D}_{2 \mathrm{cc}}$ was $\leq 65 \mathrm{~Gy}$, the rate of grade 2 bleeding was $<5.2 \%$, and the risk of proctitis was $<4.6 \%$ in previous reports. However, the incidence of rectal fistula is reported to be approximately $2.7 \%$ when a $\mathrm{D}_{2 \mathrm{cc}}<75 \mathrm{~Gy}$ is applied [22]. In our study, the mean $\mathrm{D}_{2 \mathrm{cc}}$ rectum dose was $63.10 \mathrm{~Gy}$ with T-ring application, and 74.99 Gy with T-ovoid application.

Tuncel et al. [11] performed a dosimetric comparison of ring and ovoid applicators in a model of post-operative vaginal cuff radiotherapy that did not include HR-CTVs (because of the nature of post-operative protocol). They noted a lower rectum reference dose when using ring applicators compared with ovoid applicators, which was consistent with our findings.

Levin et al. [12] in 17 patients, compared radiation delivery between ovoid and ring applicators using a 3D design as well as planning optimization for point A. Although the applicators delivered similar prescribed doses to point $\mathrm{A}$ and maintained the critical organ doses below tolerance level, the volumes treated were significantly different between types of applicator. They concluded that T-ovoid applicators may deliver too high doses to surrounding healthy tissues, while T-ring applicators may not provide enough radiation to tumor tissue. In this study, we observed that the point A dose was not correlated with the HR-CTV. The T-ovoid applicator released a greater dose laterally, particularly when the tumor volume was not defined, and only lateral points were considered, thus providing a dosimetric advantage. However, the irradiated volumes differed dramatically between applicator types and at a defined HR-CTV, the ring position helped to provide consistent coverage.

Ma et al. [13] also compared ovoid and ring dosimetry and clinical outcomes in 13 patients. Although they detected a larger isodose volume with T-ovoid application, the CTV $\mathrm{D}_{90}$ did not significantly differ between types of applicator, so that the T-ovoid irradiated a greater volume outside the CTV. The applicators were applied in different patients, emphasizing that various radiation oncologists clearly exhibited a preference for one applicator type over the other. Differences in these preferences may account for the dosimetric differences observed. In our study, we attempted to provide consistency by using both applicator types in the same patient by the same radiation oncologist, who then performed the dosimetry assessments.

The radiation doses to normal tissue volumes receiving $100 \%$ and $200 \%$ of the prescribed doses were higher with the use of T-ovoid applicator $[12,13,23]$. Thus, larger, laterally extended tumors could benefit from the T-ovoid. Consistent with the results of Tuncel et al. [11], lateral doses were higher with the use of T-ovoids. In our study, four patients who showed better dosimetry profiles with the T-ovoid applicator may have had larger or more laterally extended tumors, but the capacity of the study was insufficient to allow meaningful statistical analysis of these patients as a subgroup.

Rangarajan [23] conducted a retrospective dosimetric comparison of the T-ring and T-tandem applicators, using a data set including $100 \mathrm{CT}$ scans of patients who were treated with a dose of 8 Gy prescribed to point A. The T-ring applicator was associated with a superior dosimetry profile to the T-ovoid $\left(\mathrm{D}_{2 \mathrm{cc}}\right)$ for the rectum (4.79 Gy vs. $4.04 \mathrm{~Gy} ; p=0.003)$, sigmoid (3.29 Gy vs. $2.80 \mathrm{~Gy} ; p=0.004)$, and bladder (7.19 Gy vs. $6.75 \mathrm{~Gy}$; $p=0.19)$. It was suggested that these findings may have been due to differences in tandem length and angle, or to the absence/ presence of a rectal retractor. In our study, we found both superior organ protection for the rectum and bladder, with the same length and angled tandems. The presence of a rectal retractor in the T-ring may have accounted for good rectal protection presented by this applicator.

This study was limited by a lack of dedicated planning for the MRI. The HR-CTV was delineated based only on the post-external, T2-weighted MRI images, and adaptive treatment planning was not performed. Also, the sample size was relatively small. However, the findings of this preliminary study could serve as a basis for further studies. Future studies including more patients will be helpful for identifying which patients will likely show superior dosimetry profiles for the T-ovoid vs. T-ring applicator.

\section{Conclusions}

Although this study had some limitations, it is the first to compare the T-ring and T-ovoid applicators using a paired-sample design and a 3D technique optimized for HR-CTV. We concluded that although the ICRU 38-point A doses were higher using T-ovoid application, the HR-CTV dose coverage was better using T-ring application. Higher HR-CTV doses can be applied using T-ring application. Although some patients had better dosimetry profiles with the T-ovoid applicator, we could not perform a subgroup analysis to define their characteristics. In general, rectum and bladder doses were statistically lower under T-ring application, which could be due to the presence of a rectal retractor. Thus, with a limited number of samples, we conclude that the T-ring applicator offers superior radiation delivery and brachytherapy protection compared with the T-ovoid applicator, but larger studies comparing these applicators in the presence of rectal retractors are necessary.

\section{Disclosure}

The authors report no conflict of interest. 


\section{References}

1. Ferlay J, Soerjomataram I, Dikshit R et al. Cancer incidence and mortality worldwide: sources, methods and major patterns in GLOBOCAN 2012. Int J Cancer 2015; 136: E359-386.

2. Miglierini P, Malhaire JP, Goasduff $G$ et al. Cervix cancer brachytherapy: high dose rate. Cancer Radiother 2014; 18 . 452-457.

3. Turkish Cancer Statistic Database. Department of Cancer, Turkish Ministry of Health, 2014, pp. 16-40.

4. Shrivastava S, Mahantshetty U, Engineer R et al. Treatment and outcome in cancer cervix patients treated between 1979 and 1994: a single institutional experience. J Cancer Res Ther 2013; 9: 672-679.

5. Eifel PJ, Thoms WW Jr, Smith TL et al. The relationship between brachytherapy dose and outcome in patients with bulky endocervical tumors treated with radiation alone. Int J Radiat Oncol Biol Phys 1994; 28: 113-118.

6. Perez CA, Grigsby PW, Chao KS et al. Tumor size, irradiation dose, and long-term outcome of carcinoma of uterine cervix. Int J Radiat Oncol Biol Phys 1998; 41: 307-317.

7. Sturdza A, Pötter R, Fokdal LU et al. Image guided brachytherapy in locally advanced cervical cancer: Improved pelvic control and survival in RetroEMBRACE, a multicenter cohort study. Radiother Oncol 2016; 120: 428-433.

8. Sadeghi MH, Sina S, Mehdizadeh A et al. The effect of tandem-ovoid titanium applicator on points A, B, bladder, and rectum doses in gynecological brachytherapy using ${ }^{192} \mathrm{Ir}$. J Contemp Brachytherapy 2018; 1: 91-95.

9. Derks K, Steenhuijsen JLG, van den Berg HA et al. Impact of brachytherapy technique (2D versus $3 \mathrm{D}$ ) on outcome following radiotherapy of cervical cancer. J Contemp Brachytherapy 2018; 1: 17-25.

10. Sud S, Roth T, Jones E. Clinical analysis of speculum-based vaginal packing for high-dose-rate intracavitary tandem and ovoid brachytherapy in cervical cancer. J Contemp Brachytherapy 2018; 1: 32-39.

11. Tuncel N, Toy A, Demiral AN et al. Dosimetric comparison of ring and ovoid applicators. J BUON 2009; 14: 451-455.

12. Levin D, Menhel J, Rabin T et al. Dosimetric comparison of tandem and ovoids vs. tandem and ring for intracavitary gynecologic applications. Med Dosim 2008; 33: 315-320.

13. Ma JK, Mourad WF, Allbright $\mathrm{R}$ et al. Short term clinical outcomes of dosimetric comparison of tandem and ring versus tandem and ovoids intracavitary applicators. J Contemp Brachytherapy 2015; 7: 218-223.

14. Haie-Meder C, Pötter R, Van Limbergen E et al. Recommendations from Gynaecological(GYN) GEC-ESTRO WorkingGroup (I): Concepts and terms in 3D image based 3D treatmentplanning in cervix cancer brachytherapy with emphasison MRI assessment of GTV and CTV. Radiother Oncol 2005; 74: 235-245

15. Pötter R, Haie-Meder C, Van Limbergen E et al. Recommendations from gynaecological (GYN) GEC ESTRO working group (II): Concepts and terms in 3D image-based treatment planning in cervix cancer brachytherapy-3D dose volume parameters and aspects of 3D image-based anatomy, radiation physics, radiobiology. Radiother Oncol 2006; 78: 67-77.

16. Hellebust TP, Kirisits C, Berger D et al. Recommendations from Gynaecological (GYN) GEC-ESTRO Working Group: considerations and pitfalls in commissioning and applicator reconstruction in 3D image-based treatment planning of cervix cancer brachytherapy. Radiother Oncol 2010; 96: 153-160.

17. ICRU Report 38. Dose and Volume Specification for Reporting Intracavitary Therapy in Gynecology. Bethesda, MD: International Commission on Radiation Units and Measurements, 1985; pp. 1-20.
18. Ginsburg O, Bray F, Coleman MP et al. The global burden of women's cancers: a grand challenge in global health. Lancet 2017; 389: 847-860.

19. Schmid M, Haie-Meder C, Mahanshetty U et al. Local failures after radiochemoterapy and MR-image-guided brachytherapy in cervical cancer patients. Radiother Oncol 2017; 123: S26.

20. Tanderup K, Fokdal LU, Sturdza A et al. Effect of tumor dose, volume and overall treatment time on local control after radiochemotherapy including MRI guided brachytherapy of locally advanced cervical cancer. Radiother Oncol 2016; 120: 441-446.

21. Fokdal L, Sturdza A, Mazeron R et al. Image guided adaptive brachytherapy with combined intracavitary and interstitial technique improves the therapeutic ratio in locally advanced cervical cancer: analysis from the retroEMBRACE study. Radiother Oncol 2016; 120: 434-440.

22. Mazeron R, Fokdal LU, Kirchheiner K et al. Dose-volume effect relationships for late rectal morbidity in patients treated with chemoradiation and MRI-guided adaptive brachytherapy for locally advanced cervical cancer: results from the prospective multicenter EMBRACE study. Radiother Oncol 2016; 120: 412-419.

23. Rangarajan R. Dosimetric evaluation of image based brachytherapy using tandem ovoid and tandem ring applicators. Rep Pract Oncol Radiother 2018; 23: 57-60. 